\title{
Comparative Study of CCD \& CMOS Sensors for Image Processing
}

\author{
Kalpana Kumbhar ${ }^{1}$, Dr. Ketki P.Kshirsagar ${ }^{2}$ \\ Assistant Professor, Vishwakarma Institute of Information Technology, Pune ${ }^{1,2}$
}

\begin{abstract}
Compression is one of the most demanding of the processing steps. Still, image compression is achieved by removing spatial redundancy, while in video devices, temporal redundancy can be used to further improve the compression performance. Various image compression algorithms and coding schemes have been proposed, such as predictive coding, Discrete Cosine Transform (DCT) based compression algorithms and wavelet-based compression, Joint Photographic Experts Group (JPEG) algorithms, moving Picture Experts Group (MPEG) standards. VLSI circuit implemented image/video compression standards have been reported in the early literature Improvement in CMOS technology results in CMOS image sensor competitive to CCD image sensor in many applications. In this article, we provide a basic introduction to CMOS and CCD image-sensor technology, design and performance limits and present recent developments and future directions in this area correspondingly. CMOS technology enables the integration of image sensing and image processing, making the CMOS image sensor the optimum solution to improve the performance of the overall system. In the last several decades, image sensors integrating different on-chip compression algorithms, such as predictive coding. One of the most important advantages of CMOS image sensors over CCDs is the ability to integrate sensing with analog and digital processing down to the pixel level.
\end{abstract}

Keywords: CMOS, CCD, image sensor.

\section{INTRODUCTION}

An image sensor is one of the main building blocks in a digital imaging system such as a digital still or video camera. Fig.1 depicts a simplified block diagram of [4]. imaging system architecture.

First, the scene is focused on the image sensor using the imaging optics. Image sensors comprising a two dimensional array of pixels converts the light incident at its surface into an array of electrical signals. To perform color imaging, a color-filter array (CFA) is typically deposited in a certain pattern on top of the image sensor pixel array (Fig 4 for a typical red-green-blue Bayer CFA)[1]. Using such a filter, each pixel produces a signal corresponding to only one of the three colors, e.g., red, green, or blue. The analog pixel data (i.e., the electrical signals) are read out of the image sensor and digitized by an analog-to-digital converter (ADC). To produce a full color image, i.e., one with red, green and blue color values for each pixel, a spatial interpolation operation known as demosaicking is used. Further digital-signal processing is used to perform white balancing and color correction as well as to diminish the adverse effects of faulty pixels and imperfect optics. Finally, the image is compressed and stored in memory [2]. Other processing and control operations are also included for performing auto-focus, auto-exposure, and general camera control. The sensor used in a digital camera has a 2-D array of thousands or millions of tiny solar cells, each of which transforms the light from one small portion of the image into electrons [3]

\section{SENSORS}

\subsection{CMOS sensors}

A CMOS imaging chip is a type of active pixel sensor made using the CMOS semiconductor process shown in fig. 2. Extra circuitry next to each photo sensor converts

the light energy to a voltage. Additional circuitry on the chip may be included to convert the voltage to digital data

A CCD device, the charge is actually transported across the chip and read at one corner of the array. An analog-todigital converter turns each pixel's value into a digital value. In most CMOS devices, there are several transistors at each pixel that amplify and move the charge using more traditional wires. The CMOS approach is more flexible because each pixel can be read individually [5].

\subsection{CCD sensors}

In a CCD sensor, the light that falls on the pixels of the sensor is transferred from the chip through one output node, or only a few output nodes. The charges are converted to voltage levels, buffered, and sent out as an analog signal. This signal is then amplified and converted to numbers using an $\mathrm{A} / \mathrm{D}$-converter outside the sensor, see Fig 3. The CCD technology was developed specifically to be used in cameras, and CCD sensors have been used for more than 30 years. Traditionally, CCD sensors have had some advantages compared to CMOS sensors, such as better light sensitivity and less noise. In recent years, however, these differences have disappeared [6].

The disadvantages of CCD sensors are that they are analog components that require more electronic circuitry outside the sensor, they are more expensive to produce, and can consume up to 100 times more power than CMOS sensors. The increased power consumption can lead to heat issues in the camera, which not only impacts image quality negatively, but also increases the cost and environmental impact of the product. CCD sensors also require a higher data rate, since everything has to go through just one output amplifier, or a few output amplifiers. 


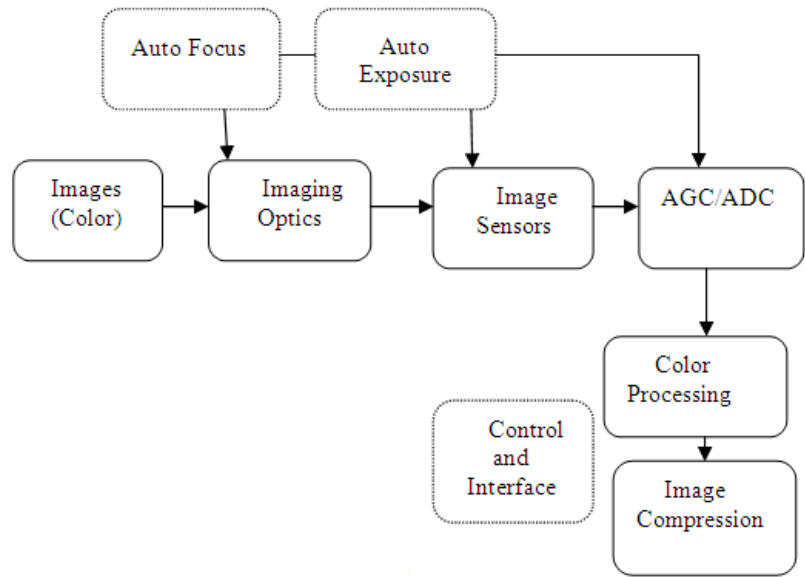

Fig 1: Imaging System Architecture.

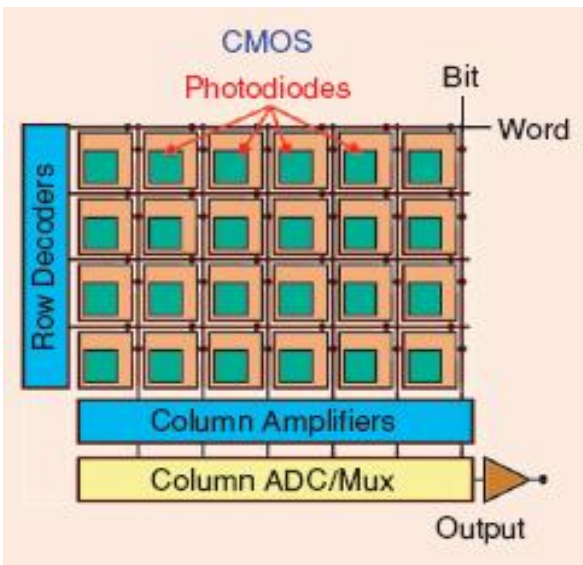

Fig 2: CMOS Sensor

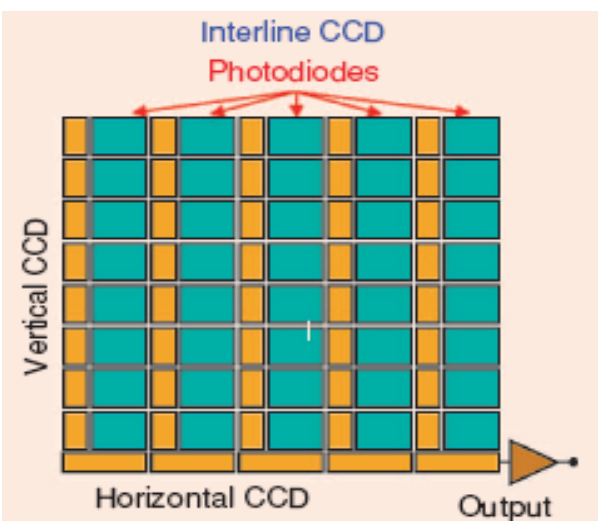

Fig.3: CCD Sensor

Each cell of a CCD image sensor is an analog device. When light strikes the chip it is held as a small electrical charge in each photo sensor. The charges are converted to voltage one pixel at a time as they are read from the chip. Additional circuitry in the camera converts the voltage into digital information[7].

\section{COLOR FILTERING}

Image sensors register the amount of light from bright to dark with no color information. Since CMOS and CCD image sensors are 'color blind', a filter in front of the sensor allows the sensor to assign color tones to each pixel. Two common color registration methods are RGB
(Red, Green, and Blue) and CMYG (Cyan, Magenta, Yellow, and Green). Red, green and blue are the primary colors that, mixed in different combinations, can produce most of the colors visible to the human eye. The Bayer array, which has alternating rows of red-green and greenblue filters, is the most common RGB color filter, see Fig.3 (left). Since the human eye is more sensitive to green than to the other two colors, the Bayer array has twice as many green color filters. This also means that with the Bayer array, the human eye can detect more detail than if the three colors were used in equal measures in the filter. Fig. 3. Bayer array color filter (left) and CMYG color filter array (right). Another way to filter or register color is to use the complementary colors - cyan, magenta, and yellow. Complementary color filters on sensors are often combined with green filters to form a CMYG color array, see Fig. 3 (right). The CMYG system generally offers higher pixel signals due to its broader spectral band pass. However, the signals must then be converted to RGB since this is used in the final image, and the conversion implies more processing and added noise. The result is that the initial gain in signal-to-noise is reduced, and the CMYG system is often not as good at presenting colors accurately. The CMYG color array is often used in interlaced CCD image sensors, whereas the RGB system primarily is used in progressive scan image sensors. For more information about interlaced CCD image sensors and progressive scan image sensors[8].
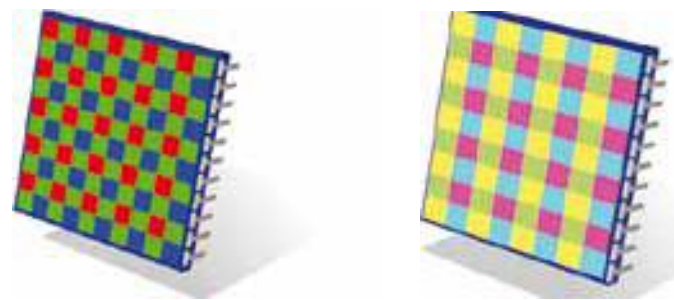

Fig. 4: Bayer array color filter and CMYG color filter array

\section{COMPARISON OF BOTH SENSORS}

\subsection{Output}

For the basic architectural reasons, output of the CCD sensors are analog while that of the CMOS sensors are digital. CMOS sensors have their own charge to voltage conversion system, signal amplification and noise elimination system [9]. This allows camera manufacturers to basically design a digital camera on a chip. CCD based camera require additional mechanisms to convert the signals to electron, then correct for noise as well as convert the voltage to digital image. CCD camera sensors are very good at capturing light. Because of the way pixels are arranged when compared to CMOS, they tend to perform a lot better in low-light situations. CMOS camera sensors also consume a lot less energy than CCD camera sensors. In fact, this energy consumption is cut down in some cases by a factor of 10 .

\subsection{Uniformity of the Signal Output}

Each pixel of a CMOS sensor has its own charge to digital bit conversion system, maintaining an uniformity in as much as image quality and exposure is difficult in 
different (but uniform for all pixels) lighting conditions. cameras on these phones. The industry demanded a sensor CCD's are much better off in this area. For a period of that is capable of being built into smaller forms. Thus time CCD's were thus preferred as they offered better CMOS systems came back into reckoning. Major response and handled different lighting conditions investments were made to make it possible to manufacture uniformly across all pixels. However, off late, better CMOS sensors cost effectively leading in their wideconversion algorithms in CMOS systems have started to close this gap and performance too has been comparable. CMOS camera sensors, which are less light-sensitive, often have to be compensated for with the introduction of ISO, which introduces noise (grain) to the image

\subsection{Antiblooming}

The same architecture, however, had an advantage when it came to correcting overexposure (in certain cases) affecting a small percentage of the sensors, without affecting the overall picture. Meaning, if a few of the pixels suffered from overexposure that can be corrected easily on a CMOS sensor (because it handles signal conversion for each pixel individually) than on a CCD sensor (which is dependent on conversion off-sensor. CMOS camera sensors have an advantage when facing a white background as CCD camera sensors can present a smearing in brightly lit objects. Newer CMOS camera sensors are becoming less sensitive to the movement of a camera as they capture images faster, though they can still present the problem in certain circumstances.

\subsection{Speed of Conversion}

CMOS sensors, in spite of their less than satisfactory results in lowlight conditions, are found to be faster in terms of image processing when compared to CCD. Since each pixel does its own conversion from analog to digital signal, they work independent to each other [10]. Additionally, the transfer of signals to the image processing engines is also done using their independent wiring, making the whole process faster. CCD sensors on the other hand have to depend off-sensor in order to convert the light signals to voltage and then transferred to the image processing engine. Each individual pixel propagates its signal to the next one and this continues till the pixel closest to the output node is reached where it is offloaded and sent to the processing engine for conversion to digital image. This results in a much slower transfer rate. Both $\mathrm{CCD}$ and $\mathrm{CMOS}$ sensors were developed roundabout the same time (1960's). However, the available manufacturing technology at that time meant that it was the CCD sensors that could be more effectively produced. Thus they were produced in greater numbers. CMOS camera sensors are cheaper and more energy 8 efficient. CCD camera sensors are more light sensitive and less prone to rolling shutter wobble.

\subsection{The Physiology of the Grid}

An open grid services architecture for distributed systems integration. Technical report, Global Grid Forum (2002). One of the major reasons why CMOS sensors are now being preferred over their CCD cousins is that they are less power hungry. Additionally, the growth in demand of on-chip camera systems which are cheaper to make and are comparable in performance to CCD powered systems is also an attributing reason. With the growth in sales of smart phones and iPhones there was need for an efficient and less power hungry sensor that could power the spread use. CCD camera sensors also have an advantage when it comes to rolling shutter wobble (also known as jellification) when an image is moved or twisted. This wobble is present on CMOS camera sensors due to the rolling shutter, while CCD camera sensors are practically immune to this issue[11].

\section{CONCLUSIONS}

CCD and CMOS sensors have different advantages, but the technology is evolving rapidly and the situation changes constantly. Hence, the best strategy for a camera manufacturer and the one that Axis Communications adheres to is to continually evaluate and test sensors for each and every camera that is developed. The question whether a chosen sensor is based on CCD or CMOS technology then becomes irrelevant. The only focus is if the sensor can be used to build a network camera which delivers the image.

In spite of the significant advances in CMOS image-sensor technology and design, the smaller pixel size and generally better low-light performance of CCDs remain as the main obstacles toward their general adoption. We expect that further scaling of CMOS image sensor technology and improvements in their imaging performance will eventually erase any remaining advantage of CCDs. More importantly, we expect that many new and very large markets for imaging devices will be created by further exploitation of the integration of sensing and processing.

\section{REFERENCES}

1. Janesick J, "Dueling detectors CMOS or CCD?" spies oe magazine 2002, 30-33.

2. Janesick J.; G. Putnam, "Developments and Applications of High Performance CCD and CMOS Imaging Arrays" Annu Rev. Nucl. Part. Sci. 2003, 53;x;x

3. O'Rourke B., "Carving out new frontiers" spies oe magazine 2002, 36-37

4. Abbas El Gamal ,Helmy Eltoukhy, “CMOS image sensors IEEE circuits \& devices magazine", may/june 2005

Axiss communication:CCD and CMOS sensor technology Kazuhiro Satu: Image processing algorithm ,Google books

Rajib Mukherjee, "CCD vs CMOS: A Comparative Analysis Of The Two Most Popular Digital Sensor Technologies”, June 12, 2014

K Hong, Ph.D.:CCD vs CMOS Sensors 2014.

Ashok Kumar, Rajiv Kumaran: 'Improvement in DPCM image Compression Technique', it SIP-2013, 18-19 Oct-2013, MumbaiIndia, pp 280-284.

10. Majid Rabbani: 'Digital Image Compression Techniques', Eastman Kodak Company, Volume TT 7, Spie Optical Engineering Press, 1995.

11. Rafel C Gonzalez, "Digital Image Processing using MATLAB". 